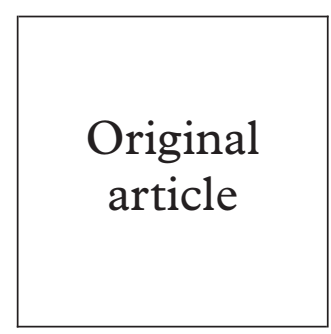

Department of Social Science and Medicine (DSSM), Imperial College School of Medicine, London $M$ Hickman

A Judd

A Renton

\section{Public Health} Laboratory Service (PHLS),

Communicable

Disease Surveillance

Centre (CDSC),

Thames

H Maguire

PHLS Communicable Disease Surveillance Centre, Colindale, London

M Catchpole

PHLS Statistics Unit, Colindale, London A Charlett

\section{Genitourinary}

Medicine Clinic, St George's Hospital

Medical School,

P Hay

Genitourinary

Medicine Clinic,

Southlands Hospital,

Worthing

A Nayagam

Correspondence to: M Hickman.

\title{
Incidence of gonorrhoea diagnosed in GUM clinics in South Thames (West) Region
}

Matthew Hickman, Ali Judd, Helen Maguire, Phil Hay, André Charlett, Mike Catchpole, Andrew Nayagam, Adrian Renton

Objectives: To describe the incidence of gonorrhoea diagnosed in genitourinary medicine (GUM) clinics in South Thames (West) between 1995 and 1996, and how it changed among population subgroups.

Settings and subjects: Cases of uncomplicated and complicated gonorrhoea diagnosed at 13 GUM clinics in the former South Thames West (STW) Regional Health Authority that reported disaggregate data to the South Thames GUM Clinic Collaborative STD Surveillance Scheme.

Methods: Annual incidence rates (per 100 000) of gonorrhoea diagnoses by sex, age group, ethnic group, area of residence, and year were calculated. Poisson regression models were used to calculate risk ratios (RR) to describe the key differences in the variation of gonorrhoea cases by these variables. Relative differences in the incidence of diagnosed gonorrhoea between 1995 and 1996 were investigated by including an interaction between year and the other variables (age group, sex, ethnic group, region) and testing whether any were significant using a likelihood ratio test.

Results: Area of residence, sex, age group, and ethnic group were key predictors of the rates of diagnosed gonorrhoea. The risk ratio for gonorrhoea (after adjustment for the other variables) was: 13 times higher among blacks than the white population; twice as high in inner London compared with outer London; and three times lower in the "shire" region compared with outer London. The rate of diagnosed gonorrhoea was significantly higher in the black population in the shire region than the inner London white population. The rate of gonorrhoea diagnosed by GUM clinics from 1995 to 1996 almost doubled in the white population aged 15-44 years, from 16 cases per 100000 to 30 cases per 100000 (adjusted RR 2.0, 95\% CI 1.6 to 2.4), whereas increased rates in the black and Asian/other ethnic groups were not statistically significant (adjusted RR 1.1, 95\% CI 0.9 to 1.4 ; and 1.4 , 95\% CI 0.7 to 2.7 respectively).

Conclusion: The observed increase in gonorrhoea between 1995 and 1996 occurred mostly among heterosexual white men and women. Overall, the rates of gonorrhoea among young people, especially in the black population and in inner London represent a significant public health problem that may merit further targeted interventions, the effectiveness of which could be monitored through further development of routine surveillance data.

(Sex Transm Inf 1999;75:306-311)

Keywords: gonorrhoea; genitourinary medicine clinics; South Thames (West) Region

\section{Introduction}

In 1997 the Public Health Laboratory Service (PHLS) Communicable Disease Surveillance Centre (CDSC) reported that the incidence of gonorrhoea in England and Wales rose between 1995 and 1996, based on data from routine KC60 reporting by genitourinary medicine (GUM) clinics. ${ }^{1}$ Apart from a small upturn reported at the beginning of the 1990s which was short lived and attributed to a resurgence of gonorrhoea among homosexual men, ${ }^{2-4}$ this was the second reversal in a more or less progressive decline in gonorrhoea incidence since the mid-1980s. The rise noted between 1995 and 1996 is notable because it concerns cases of gonorrhoea diagnosed among all age and sex groups within GUM clinics, and also because of its relevance as a marker of the sexual health of the population and increased risk of HIV infection. ${ }^{5}$ Indeed, the decline of gonorrhoea infections among homosexual men was considered by many to be corroborating evidence of a significant change in sexual behaviour responsible for the reduction in the incidence of sexually acquired HIV infection in the United Kingdom, ${ }^{6}$ though its usefulness as a marker of HIV has been questioned. $^{7}$

The epidemiology of gonorrhoea has been shown to vary widely between different population subgroups, with much higher rates found among teenage women and young men, among the black population, and in urban inner city areas in England. ${ }^{89}$ Similar patterns of incidence have been reported in the United States. ${ }^{1011}$ It is not clear why some ethnic groups have higher rates, and although gonorrhoea has been associated with poverty, ethnic differences remain after adjustment for socioeconomic confounding. ${ }^{9} 12$

In this study we describe the patterns of gonorrhoea diagnosed in GUM clinics between 1995 and 1996 in a region of southern England, the former South Thames West Regional Health Authority (STW). STW covers three geographical areas: inner London, outer London, and "shire county" with a combined population of 3.2 million.

\section{Methods}

ASCERTAINMENT OF CASES

Thirteen departments of GUM in STW routinely contribute disaggregate data on all 
STD diagnoses (KC60) to the South Thames GUM Clinic Collaborative STD Surveillance Scheme. ${ }^{13}$ Cases of uncomplicated and complicated gonorrhoea infection (KC60 codes B1, B2, B5) diagnosed between 1 January 1995 and 31 December 1996 were extracted. Fifteen duplicate records, defined as a second record of gonorrhoea within the same quarter in the same patient at the same GUM clinic, were removed. Patients with another diagnosis of gonorrhoea after 3 months were assumed to have acquired a new infection, and were included as cases. Possible repeat infections within and between clinics were identified by matching cases using soundex code, year of birth, and sex.

The patients' postcodes of residence were used to allocate cases into three areas of residence based on local government boundaries: inner London, outer London, and shire. All diagnosed cases were included in our analyses under the assumption that migration out of STW was at least equal or possibly greater than migration into STW for treatment. This was supported by data from a local GUM database in a neighbouring health authority ( $\mathrm{P}$ Trail, personal communication). Cases without a postcode of residence were excluded from regional analyses but included in the overall totals.

DENOMINATOR POPULATIONS

Office for National Statistics population estimates from the 1991 census, adjusted for underenumeration, ${ }^{14}$ were used to estimate the population at risk of gonorrhoea infection stratified by area of residence in STW, sex, age group, and ethnic group. Ethnic group was further collapsed into three categories: "white," "black" (black Caribbean, black African, and black other), and "Asian/other" (Indian, Pakistani, Bangladeshi, Chinese, and other). Population estimates from 1991 were used because subsequent population estimates did not provide a breakdown by ethnic group. We conducted a sensitivity analysis to compare the rates and findings of the statistical analysis using 1996 population totals instead of the 1991 estimates. The 1996 estimates were aggregated into ethnic groups according to the proportions recorded in 1991 and estimates of the relative growth of different ethnic populations in Greater London (London Research Centre, unpublished).

\section{STATISTICAL ANALYSIS}

Annual rates (per 100 000) of gonorrhoea diagnosed in GUM clinics by sex, age group, ethnic group, and area of residence were calculated with $95 \%$ confidence intervals (CI). If no cases were observed within a specific stratum (for example, black males aged 15-19 in shire), exact confidence intervals, assuming a Poisson distribution, were calculated (data not shown, available on request). Poisson regression models, with the number of cases of gonorrhoea expressed as a rate as the dependent variable and age group, sex, ethnic group, area of residence, and year as predictors, were used to examine differences between the subgroups, which are presented as a series of risk ratios (RR).

Interactions were sought between the variables for two purposes. Firstly, in order to provide a better description of the variation in rates of diagnosis, interactions were tested between age group, sex, ethnic group and area of residence. Interaction terms were included in the overall model if the $p$ value from the likelihood ratio test (LRT) was less than $0.01 .^{15}$ It has been argued that, whenever possible, the correct approach is to use a single model to describe the results rather than to stratify the data and carry out separate analyses for each strata (for example, for males and females). ${ }^{16}$ This means, however, that variables that interact cannot be reported independently of each other, and that their risk ratios need to be recalculated. For example, for age and sex the baseline was males aged 30-44, and risk ratios were calculated for all other sex and age categories against the baseline, from males aged 25-29 to females aged 15-19.

Secondly, the null hypothesis that the observed increase in gonorrhoea between 1995 and 1996 was the same for the main variables was explored by fitting an interaction between year and the other variables (that is, year*age group, year ${ }^{\star}$ sex, year*ethnic group and year*area of residence). The overall fit of the model with and without interactions between year and the other variables was tested using the LRT, and if significant included in the final model of the data. Risk ratios were recalculated for any interaction terms as above to show differences in the rate of diagnosis between 1995 and 1996.

The statistical software package STATA 5.0 (Stata Corp, College Station, TX, USA) was used for all analyses.

\section{Results}

SAMPLE CHARACTERISTICS

In total, 1056 cases of gonorrhoea among individuals aged 15-44 years were diagnosed from

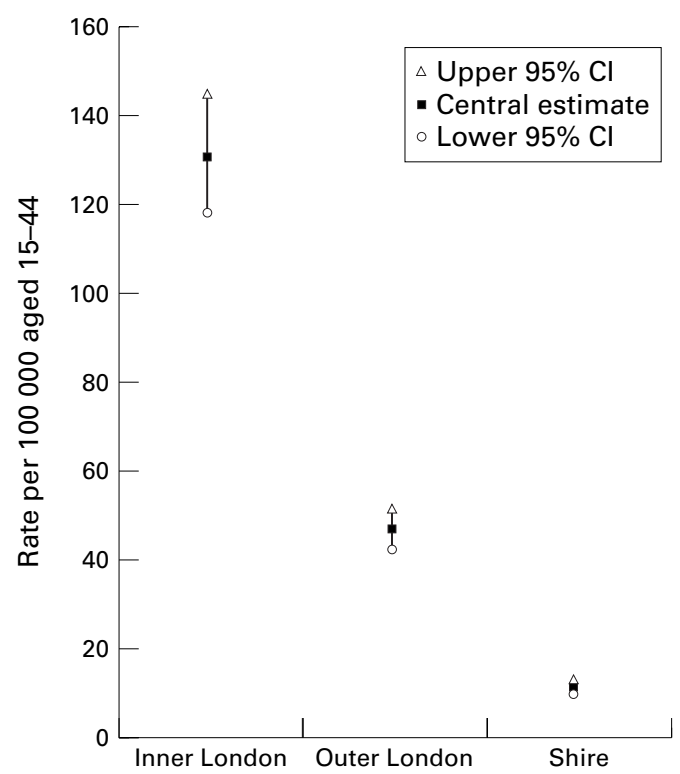

Figure 1 Rate of gonorrhoea diagnosed in GUM clinics by area of residence (South Thames West 1995-6). 
Table 1 Adjusted ${ }^{*}$ risk ratios with 95\% confidence intervals for the effects of area of residence, sex, age, ethnic group and year on the rate of gonorrhoea diagnosed in GUM clinics (South Thames West, 1995-6)

\begin{tabular}{|c|c|c|c|c|c|}
\hline Variable & $\begin{array}{l}\text { Risk } \\
\text { ratio }\end{array}$ & $95 \% C I$ & Variable & $\begin{array}{l}\text { Risk } \\
\text { ratio }\end{array}$ & $95 \% C I$ \\
\hline \multicolumn{6}{|l|}{ Region: } \\
\hline Inner & 1.98 & 1.70 to 2.30 & & & \\
\hline Outer & $1 \dagger$ & & & & \\
\hline Shire & 0.31 & 0.26 to 0.38 & & & \\
\hline \multicolumn{6}{|c|}{ Sex and age group: } \\
\hline Males & & & Females & & \\
\hline $15-19$ & 1.27 & 0.94 to 1.70 & $15-19$ & 2.28 & 1.79 to 2.90 \\
\hline $20-24$ & 2.21 & 1.77 to 2.77 & $20-24$ & 1.55 & 1.22 to 1.97 \\
\hline $25-29$ & 2.06 & 1.66 to 2.56 & $25-29$ & 0.69 & 0.52 to 0.93 \\
\hline $30-44$ & $1 \dagger$ & & $30-44$ & 0.20 & 0.14 to 0.28 \\
\hline \multicolumn{6}{|c|}{ Year and ethnic group: } \\
\hline 1995 & & & 1996 & & \\
\hline White & $1 \dagger$ & & White & 1.96 & 1.63 to 2.36 \\
\hline Black & 13.66 & 10.88 to 17.16 & Black & 15.69 & 12.59 to 19.56 \\
\hline Asian/other & 1.03 & 0.61 to 1.75 & Asian/other & 1.44 & 0.91 to 2.27 \\
\hline \multicolumn{6}{|l|}{$1995 v 1996$} \\
\hline White‡ & 1.96 & 1.63 to 2.36 & & & \\
\hline Black $\ddagger$ & 1.15 & 0.92 to 1.43 & & & \\
\hline Asian/other $\neq$ & 1.40 & 0.72 to 2.72 & & & \\
\hline
\end{tabular}

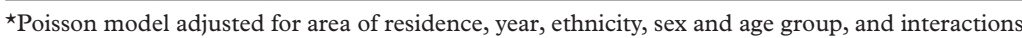
between ethnicity and year, and sex and age group.

†Baseline group.

$\ddagger$ Baseline group is the $1995 \mathrm{RR}$ for each ethnic group separately

clinics in STW, of which 429 (41\%) were diagnosed in 1995 and 627 (59\%) in 1996. There were $660(63.5 \%)$ diagnoses among males and $396(37.5 \%)$ among females. Data were missing on ethnic group for $12 \%$ (126) of cases, and on area of residence for $13 \%$ (137) of cases. Cases with missing data were distributed evenly by clinic, age group, and sex. Approximately 59\% (548) of cases were classified as white, and $38 \%$ (347) were residents of inner London. Gonorrhoea cases in women were on average $4(95 \%$ CI 3.3 to 4.7$)$ years younger than cases among men (22.7 (SD 5.9) versus 26.8 (5.5) respectively). There were no significant differences in the proportion of gonorrhoea cases in males and females by ethnic group, area of residence, or year of diagnosis. $^{4}$

MODE OF ACQUISITION

One hundred and twenty three (19\%) diagnoses among males were reported to have been

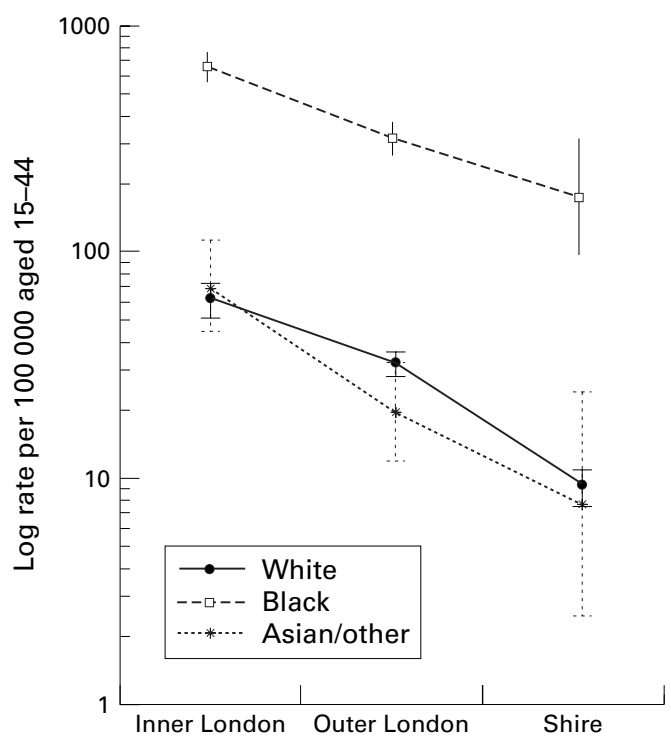

Figure 2 Rates of gonorrhoea diagnosed in GUM clinics by ethnic group and area of residence (South Thames West, 1995-6). acquired homosexually, of which 58 (22\%) were diagnosed in 1995 and 65 (16\%) in 1996, with the majority occurring among white men. The proportion of gonorrhoea infections reported as homosexually acquired in white men declined significantly from $51 / 115(44 \%)$ in 1995 to $54 / 216(25 \%)$ in $1996\left(\chi^{2} 10.1,1 \mathrm{df}\right.$, $\mathrm{p}<0.001)$. If all the cases among men $(75)$ where ethnic group was not recorded were coded as white, the fall in the proportion of homosexually acquired gonorrhoea between 1995 and 1996 would narrow but remain significant (that is, $38 \%$ versus $25 \% ; \chi^{2} 7.0,1$ df, $p=0.003)$.

\section{REPEAT INFECTIONS}

We estimated that the 1056 cases of gonorrhoea occurred among 959 patients, of whom, over the 2 year period 879 (92\%) presented with one infection, $68(7 \%)$ had two occurrences, eight patients had three occurrences, and four patients had four or more occurrences. The proportion of patients with repeat infections did not change significantly between 1995 and 1996 (9\% and $8 \%$ respectively, $\chi^{2}$ $0.75,1 \mathrm{df}, \mathrm{p}=0.4)$.

RATE OF DIAGNOSED GONORRHOEA

Overall, the rate of gonorrhoea diagnosed by GUM clinics in $1995 / 96$ was 41.2 cases per 100000 (95\% CI 38.8 to 43.7 ). This varied by age group, sex, ethnic group, area of residence, and year of diagnosis (full data not shown, available on request). In inner London the diagnosis rate was 130.6 per 100000 (95\% CI 117.5 to 145.1 ) compared with 46.6 per 100000 (95\% CI 42.2 to 51.3) in outer London, and 11.7 (95\% CI 10.0 to 13.6) in shire (fig 1). The rate of gonorrhoea diagnosed in the black ethnic group was 465.1 cases per 100000 (95\% CI 418.6 to 516.8) compared with 23.3 cases per 100000 (95\% CI 21.5 to $25.4)$ in whites and 25.3 cases per 100000 (95\% CI 18.3 to 35.1 ) in the Asian/other ethnic group. The rate for females increased from 25.6 cases per 100000 (95\% CI 21.9 to 29.8 ) in 1995 , to 35.8 cases per 100000 (95\% CI 31.5 to 40.7 ) in 1996; and for males from 41.5 cases per 100000 (95\% CI 36.8 to 46.8 ) in 1995, to 62.2 cases per 100000 (95\% CI 56.4 to 68.6) in 1996. Peak annual incidence, combining the 2 years, occurred in inner London among young black females aged 15-19 and black males aged 20-24 for whom the rates were over $1 \%$ (that is, 1376 per $10000095 \%$ CI 956 to 1980; and 1091 per $10000095 \%$ CI 771 to 1542 respectively).

Poisson regression models suggested that age group, sex, ethnic group, area of residence, and year all exerted significant effects on the rates of diagnosed gonorrhoea in STW, with significant interactions also between age group and sex (LRT $\mathrm{p}<0.0001)$, and ethnic group and year (LRT $p=0.0012$ ). Table 1 shows the adjusted risk ratios (RR) derived from the overall model for these factors, the pattern of which are further illustrated using the unadjusted rates in figures 1-4. 


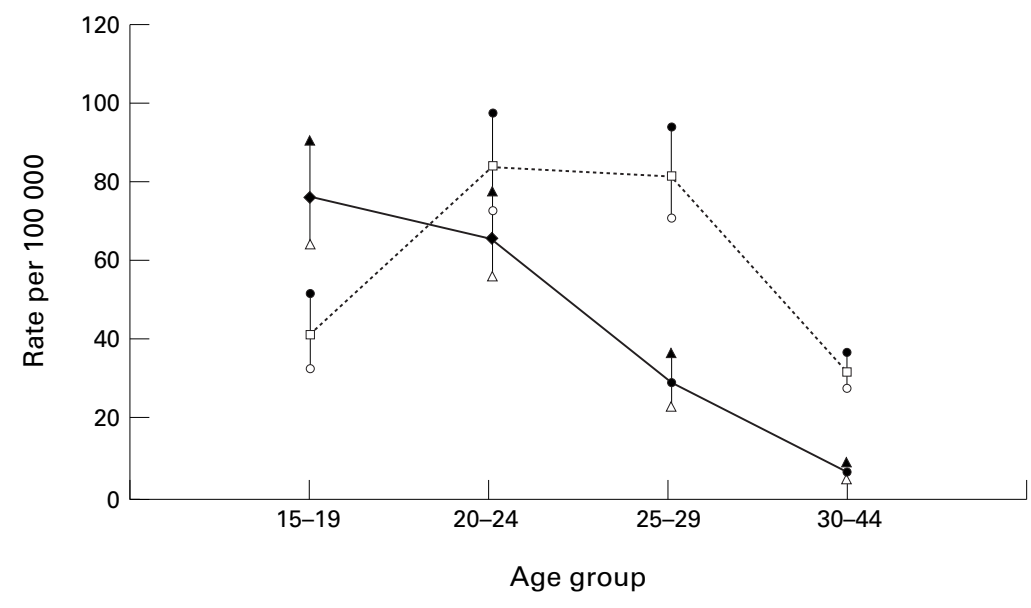

\begin{tabular}{lll}
\hline - Upper $\mathrm{Cl}$ females $\longrightarrow$ Females central estimate & $\Delta$ Lower $\mathrm{Cl}$ females \\
- Upper $\mathrm{Cl}$ males & $\cdots-.-\cdots$ Males central estimate & $\circ$ Lower $\mathrm{Cl}$ males
\end{tabular}

Figure 3 Rates of gonorrhoea diagnosed in GUM by age group and sex (South Thames West, 1995-6).

AREA OF RESIDENCE AND ETHNIC GROUP

Figure 1 shows the crude rates of gonorrhoea by area of residence. After adjustment for age group, sex, ethnic group, and year, the rate of gonorrhoea in inner London was nearly twice as high (RR 1.97 95\% CI 1.7 to 2.3 ) as outer London, and the rate in shire was three times lower (RR $0.395 \%$ CI 0.3 to 0.4 ) than outer London (table 1). This relation was independent of ethnic group, as indicated by figure 2 where the lines linking ethnic group and area of residence are approximately parallel. Figure 2 also shows that the rate of gonorrhoea in the black population resident in the shires (173.4 per $10000095 \%$ CI 96 to 313) was significantly higher than in the white population in inner London (62.4 per $10000095 \%$ CI 53 to 73) $\left(\chi^{2} 11.6,1 \mathrm{df}, \mathrm{p}=0.001\right)$

AGE AND SEX

Figure 3 shows that the pattern of rates of gonorrhoea by age group were different for males and females, or in other words that interaction was detected between age group and sex. In males the rates were higher for those aged 20-24 and 25-29, and lower for younger (1519 ) and older (30-44) groups, whereas for females the rates decreased with increasing age. Thus, young females aged 15-19 (76.7 per $100000)$ had a significantly higher incidence compared with males aged 15-19 (41.4 per $100000)\left(\chi^{2} 18.4,1 \mathrm{df}, \mathrm{p}<0.001\right)$, in contrast with the older age groups where the rates were higher for males. Because of this interaction, the risk ratios (after adjustment for ethnic group, area of residence, and year) for sex and age group together are presented in table 1 . Compared with the baseline rate in males aged 30-44, males aged $20-24$ and 25-29 had double the risk of acquiring gonorrhoea and being diagnosed (RR 2.2 95\% CI 1.8 to 2.8 ; and RR $2.195 \%$ CI 1.7 to 2.6 respectively), with the risk being approximately the same as the baseline in males aged 15-19 (RR 1.3 95\% CI 0.9 to 1.7). Among women the risk of gonorrhoea was significantly lower among those aged 30-44 and 25-29 (RR 0.2 95\% CI 0.1 to 0.3; and RR 0.7 95\% CI 0.5 to 0.9 respectively), but between one and a half and twice as high for females aged 20-24 and 15-19 (RR 1.5 95\% CI 1.2 to 2.0 ; and RR $2.395 \%$ CI 1.8 to 2.9 respectively) compared with males aged 30-44 (table 1).

ETHNIC GROUP AND YEAR

Figure 4 shows that the crude rates of gonorrhoea among black ethnic groups were considerably higher than for the white population. The largest relative increase in the rates of diagnosed gonorrhoea from 1995 to 1996, however, occurred in the white population, from 16.4 cases per 100000 (94\% CI 14.3 to $18.9)$ to 30.2 (95\% CI 27.3 to 33.6 ) respectively. Since year and ethnic group interacted, the RRs for these factors (adjusted for age group, sex, and area of residence) are reported together in table 1. After adjustment, the risk of gonorrhoea in the black ethnic group in 1995 was 13.7 (95\% CI 10.9 to 17.2 ) times higher than that for whites in 1995, which increased slightly to 15.6 (95\% CI 12.6 to 19.7) in 1996, again compared with whites in 1995 . The RRs for the Asian/other ethnic group were not significantly different from the baseline in either 1995 or 1996.

Overall, between 1995 and 1996, the risk of gonorrhoea doubled for the white ethnic group (adjusted RR 1.96, 95\% CI 1.6 to 2.4 ), but did not increase significantly in either the black ( $R R=1.15,95 \%$ CI 0.9 to 1.4 ) or Asian/other ( $\mathrm{RR}=1.40,95 \%$ CI 0.7 to 2.7 ) ethnic groups (table 1). Year did not interact significantly with any other variables.

SENSITIVITY ANALYSIS

To assess the possible magnitude of biases the population rates of diagnosed gonorrhoea and the Poisson regression models were recalcu-

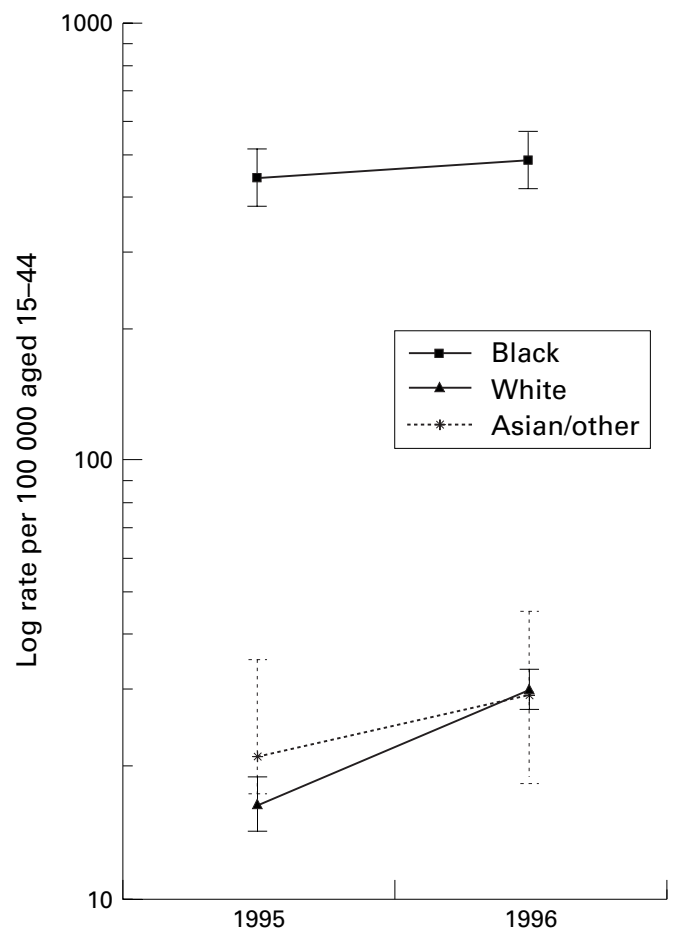

Figure 4 Rates of gonorrhoea diagnosed in GUM by ethnic group and year (South Thames West, 1995-6). 
lated using the 1996 population totals, allocated to ethnic groups. The use of 1991 population denominators instead of 1996 population estimates may have led to higher estimates of gonorrhoea incidence, although in the majority of subgroups the difference was marginal and the 95\% confidence intervals largely overlapped. Potentially, there were larger differences in inner London and for estimates for the black population (though the latter were a great deal more uncertain because of the unavailability of official ethnic specific estimates for 1996). For example, comparing estimates derived using the 1991 and our adjusted 1996 population estimates, the $95 \%$ confidence intervals for the rate of gonorrhoea diagnosed within GUM clinics were: (a) for women 32-41 per 100000 compared with 30-39 per 100 000; (b) for inner London 118-145 per 100000 compared with $110-135$ per 100000 ; and (c) for the black ethnic group 419-517 per 100000 compared with 351-433 per 100000 . However, despite a potential narrowing of the gap between white and black ethnic groups using adjusted 1996 population estimates, the order of magnitude and significance of the risk ratios presented in table 1 were not diminished.

\section{Discussion}

The figures and regression analysis presented here provide an epidemiological description of the rate of gonorrhoea diagnosed in GUM clinics in STW, and whether the observed increase between 1995 and 1996 occurred equally among different subgroups of the population. Several biases potentially affect the estimates of the rates of gonorrhoea diagnosed within GUM clinics, and their relation to the underlying incidence of gonorrhoea. Firstly, 1991 census data were used because of the lack of accurate and current population estimates by ethnic group. A sensitivity analysis suggested that this might slightly exaggerate the population rates, and differences between the black and white ethnic groups, though not sufficiently to change the scale of any of the differences. Secondly, missing data on ethnic group and area of residence resulted in some underestimation of the rates of diagnosed gonorrhoea in subgroups of the population, although because there was no evidence of bias in the distribution of missing cases this would not affect estimation of the differences in risk. Thirdly, only cases of gonorrhoea diagnosed in GUM clinics in STW were included, thereby underestimating the true incidence of gonorrhoea in the population by missing asymptomatic cases and those treated and/or diagnosed in general practice and other settings. Fourthly, we simplified the assumptions of gonorrhoea occurrence by using a Poisson model to describe and estimate differences in our data, which may not be entirely true for repeat cases. Clearly, like most observational studies care needs to be taken in interpreting the findings in the light of potential bias. We believe, however, that the biases mostly affect estimates of the incidence of gonorrhoea and not the risk ratios described in table 1; and that the scale of the estimated risks are such that the overall findings are unlikely to be changed if more bias could be taken into account.

In South Thames West, the increase in the incidence of diagnosed gonorrhoea from 1995 to 1996 was confined largely to the white population, for whom the rate of gonorrhoea among those aged 15-44 almost doubled. The adjusted risk ratio of gonorrhoea in the black and Asian/other ethnic groups did not change significantly. The increase in gonorrhoea infections was not due to any increase in "repeat" infections. It also appeared to occur almost entirely among the heterosexual population (men and women), as the number of infections acquired homosexually was unchanged and the proportion fell. This is in contrast with the previous alert of a rise in gonorrhoea which was attributed to homosexual men..$^{2-4}$ The reasons for such a differential increase, however, are not as clear. It may be due to an increase in risk behaviour among white heterosexuals, a change in their sexual mixing patterns, or simply a blip given that the rate of gonorrhoea has fallen considerably over the past decade. Further studies are required to test whether this picture is true for the rest of England and Wales and to identify the reasons for the differential increase, and further surveillance data over an extended time period are needed to show whether the rise persists or is short lived.

Our analyses enhance the description of gonorrhoea epidemiology provided by routine STD statistics, and confirm the detailed work by Low et al for Lambeth, Southwark and Lewisham, ${ }^{9}$ and Lacey et al for Leeds. ${ }^{8}$ Area of residence, sex, age group, and ethnic group were found to be key predictors of the rates of gonorrhoea diagnosed in GUM clinics in STW. The incidence of gonorrhoea (after adjustment) was estimated to be over 13 times higher among the black ethnic group than the white population, and twice as high in inner London and three times lower in shire than outer London. Moreover, our findings suggest that the rate of diagnosed gonorrhoea in the black population in the shires was higher than the rate of gonorrhoea in whites in inner London.

The high rates of gonorrhoea among the black population in inner London in our study were comparable with the studies in Leeds in $1989-93(1.6-2.1 \%$ in men and $0.5-0.7 \%$ in women) and in Lambeth, Southwark and Lewisham in 1994-5 (0.9-1.1\% in men and $0.7-1.1 \%$ in women). ${ }^{89}$ These previous studies attempted to adjust for the effects of deprivation in small geographical areas. Deprivation was measured ecologically on the basis of area of residence, and explained little of the substantial differences between ethnic groups. Our analyses also suggest that the incidence of gonorrhoea can vary across large geographical areas for blacks and whites without altering the essential inequality between the ethnic groups (see fig 2). In order to confirm these findings, however, further analyses are required covering a larger geographical area, such as the whole of the south east, as the black population in the shires within STW was small. Furthermore, there is an urgent need for more analytic stud- 
ies that collect risk behaviour and deprivation data on an individual basis to assess properly their contribution to the dynamics and relation between gonorrhoea incidence and ethnicity. ${ }^{17}$

Notwithstanding the increase among the white heterosexual population, the major public health problems are the rates of gonorrhoea diagnosed in GUM clinics among young people, especially in the black population and in inner London, which may merit further targeted prevention initiatives. Moreover, the rates of gonorrhoea are sufficiently large to enable the monitoring of the effectiveness of prevention initiatives through routine surveillance data. The proportion of cases among homosexual men was also larger than would be expected given estimates of the proportion of the population that are homosexual (that is, from $5 \%$ to $11 \%$ in inner London and lower elsewhere, compared with nearly $20 \%$ of diagnosed gonorrhoea cases among males acquired homosexually). ${ }^{18}$ Finally, these analyses demonstrate the value of changing the routine surveillance of sexually transmitted infections from a manual paper based system to an electronic system that allows better and more detailed descriptions of the epidemiology of gonorrhoea and other STIs. ${ }^{19}$

We thank the GUM consultants in South Thames West who contributed data to the South Thames GUM clinic collaborative STD Surveillance Scheme. We thank Maria Griffin (DSSM, ICSM) for extracting the data in a form that we could analyse; and Peter Madden for checking the statistical analysis analyse; and Peter Madden for checking the statistical analysis (DSSM, ICSM). We also thank Dr Tony Swann (PHLS Statistics Unit) for his helpful comments on an earlier draft. Finally, we thank the former North Thames Regional Office which
funded the Surveillance and Commissioning Information funded the Surveillance and Commissioning Information System which allowed the analysis to be carried out, a
contributed to the funding of the system in South Thames. contributed to the funding of the system in South Thames.
Contributors: MH initiated the study and, with AJ, carried out Contributors: $\mathrm{MH}$ initiated the study and, with AJ, carried out
the analysis and wrote the first draft; $\mathrm{HM}, \mathrm{PH}$, and AN were the analysis and wrote the first draft; $\mathrm{HM}, \mathrm{PH}$, and $\mathrm{AN}$ were
responsible for running the surveillance system, commenting on the study design, and editing the paper; AC designed the statistical methods for analysing the data, and edited the methods and results section; AR and MC made significant contributions to writing the paper and interpretation of the findings.

1 The incidence of gonorrhoea in England and Wales is rising. CDR Weekly 1997;7:217-20.

2 Evans B, Catchpole M, Heptonstall J, et al. Sexually transmitted diseases and HIV-1 infection among homosexual men in England and Wales. BMF 1993;306:426-8.

3 Waugh MA. Resurgent gonorrhoea in homosexual men. Lancet 1991;337:375.

4 Riley VC. Resurgent gonorrhoea in homosexual men. Lancet 1991;337:183.

5 Renton AM, Whittaker L. Using STD occurrence to monitor AIDS prevention. Soc Sci Med 1994;38:1153-65.

6 Johnson AM, Gill ON. Evidence for recent changes in sexual behaviour in homosexual men in England and Wales. Phil Trans R Soc Lond (Biol) 1989;325:153-61.

7 Adler MW. Sexual health - a health of the nation failure. BMF 1997;314:1743-7.

8 Lacey CJN, Merrick DW, Bensely DC, et al. Analysis of the sociodemography of gonorrhoea in Leeds, 1989-93. BMF 1997;314:1715-8.

9 Low N, Daker-White G, Barlow D, et al. Gonorrhoea in inner London: results of a cross sectional study. BMF 1997; 314: 1719-23.

10 Rothenberg RB. The geography of gonorrhoea. Empirical demonstration of core group transmission. Am f Epidemiol 1983;117:688-94.

11 Rice RJ, Rogerts PL, Handsfield $\mathrm{HH}$, et al. Sociodemographic distribution of gonorrhoea incidence: implications for prevention and behavioural research. $A m \mathcal{F}$ Public Health 1991;81:1252-8.

12 Moran JS, Aral SO, Jenkins WC, et al. The impact of sexually transmitted diseases on minority populations. Public Health Rep 1989;104:560-5.

13 Maguire H, Smith I, Nayagam A. Gonorrhoea and ethnicity-collaborative surveillance system is a model for ethnicity-collaborative surveillanc
the future. $B M \mathcal{F} 1997 ; 315: 1160$.

14 Office for Population Censuses and Surveys, General Registrar Office Scotland. 1991 Census user guide 58: undercoverage in Great Britain. London: OPCS, 1994.

15 Clayton D, Hills M. Statistical models in epidemiology. Oxford: OUP, 1996:100-9.

16 Altman D. Practical statistics for medical research. London: Chapman and Hall, 1991:466-7.

17 Ross MW. Race and ethnicity in STD analyses. Sex Transm Inf 1998;74:2-3.

18 Wadsworth J, Hickman M, Johnson AM, et al. Geographic variation in sexual behaviour in Britain-implications for STD epidemiology and sexual health promotion. AIDS STD epidemiol

19 Catchpole M, W Harris, Hickman M, et al. Surveillance of STIs: fit for purpose. Int $\mathcal{F} S T D$ AIDS (in press) 